Final Report:

DOE Grant Number DE-FG02-99ER54533

\title{
CURRENT PROFILE AND MAGNETIC STRUCTURE MEASUREMENTS THROUGH TANGENTIAL SOFT X-RAY IMAGING IN COMPACT TORI
}

For the period Apr. 15, 1999 thru Apr. 15, 2003

Raymond J. Fonck

University of Wisconsin-Madison

rjfonck@wisc.edu

(608) 263-779 


\section{Overview}

This project was focused on the design, fabrication, and validation of a tangentially imaging soft X-ray (SXR) camera diagnostic for determining the current distribution in strongly shaped toroidal magnetically confined plasmas such as those found in spherical tori or advanced tokamaks. It included the development of both an appropriate imaging SXR camera and image analysis techniques necessary to deduce the plasma shape and current distribution. The basic camera concept consists of a tangentially viewing pinhole imaging system with thin-film SXR filters, a scintillator screen to provide SXR to visible conversion, a fast shuttering system, and an sensitive visible camera imaging device. The analysis approach consists of integrating the 2-D SXR image data into a Grad-Shafranov toroidal equilibrium solver code to provide strong constraints on the deduced plasma current and pressure profiles. The highlights of the project include: 1) construction of a completely new magnetic equilibrium reconstruction code with an integrated nonlinear fitting routine for incorporation of the SXR image data into plasma reconstruction; 2) sensitivity tests of two imaging approaches to reconstructing the plasma current profile; 3) determination of required signal-to-noise ratio for accurate reconstruction of the plasma q-profile; 4) development and tests of two types of soft X-ray imaging camera systems; 5) demonstration of performance by measuring plasma current profiles in the Pegasus toroidal plasma; and 6) design and noise analysis for a 3rd-generation tangential camera system using direct illumination of a CCD sensor.

This diagnostic exploits the fact that the cross-sectional shape of a tokamak-like toroidal plasma is dependent on the plasma current profile. This is especially true for strongly shaped plasmas and even more pronounced on low-aspect ratio devices such as NSTX and Pegasus. Measurement of the internal shape of the poloidal flux surfaces is obtained by imaging the plasma cross-section in the soft X-ray spectral region, assuming that the electron temperature profile is tightly coupled to the flux surface. When no strong MHD activity exists, the plasma SXR emission is toroidally symmetric, and tangential imaging allows ready sampling of plasmas of arbitrary cross-section.

The measures of the flux surface shape are used as added internal constraints on reconstruction of the plasma magnetic equilibrium from magnetic equilibrium codes such as EFIT. Such measurements complement the usual external magnetics measurements and strongly constrain the internal current and possibly pressure profiles. Alone, the external magnetics alone are insufficient to determine the internal plasma profiles with any accuracy of interest. However, combining the flux surface shape constraints from SXR imaging with the external magnetics does in principle provide enough data to accurately reproduce both the internal and external plasma equilibrium parameters. In this sense, the SXR imaging plays the same role as other current profile measurements, such as polarimetry or motional Stark Effect (MSE) spectroscopy, in constraining the deduced equilibrium.

\section{A) Analysis Algorithm Development \& Diagnostic Modeling}

The development of the necessary data analysis code to allow use of the 2-D SXR images to determine the plasma current and possibly pressure profiles was a large component of this effort. To that end, we developed a completely new equilibrium code which incorporates a Grad-Shafranov solver into a nonlinear least squares fitting routine to simultaneously fit the magnetics data and the SXR image. This approach allowed ready inclusion of spline profiles for the plasma functions, and proved to be much more robust in converging to a best fit. On the negative side, the execution time is considerably longer than more traditional equilibrium codes (e.g., EFIT or Tokamac).

Model SXR images were successfully integrated into this new equilibrium and fitting code, and a wide range of tests were performed to examine how well the SXR image does constrain the fitted equilibrium. A forward modeling algorithm was integrated into the equilibrium code to use the SXR image contours to constrain the fit by using the measured midplane intensity distribution to map the emissivity to the flux surface deduced by the Grad-Shafranov solver. This map was then used to 
generate a reconstructed 2-D model image to compare to the measured image. The plasma pressure and current profiles were then adjusted to provide a best fit to the masured image intensity contours.

Two tangential imaging approaches were tested: 1) an arrangement of vertically spaced tangentially-viewing horizontal arrays of SXR diode detectors (i.e., a series of 1-D imaging detector arrays); and 2) a fully 2-D imaging system which views the plasma tangentially centered on the midplane. It was found that the first approach offers higher accuracy but requires very precise calibration and presents challenging hardware and electronics restrictions due to the need for extensive internal hardware or liberal external port access. In contrast, the second approach is less complex in its execution but its analysis is more complex and numerically intensive.

Using model images from model equiliria, a detailed evaluation of the sensitivity of the fitted plasma profiles to this added constraint of internal SXR shape data was performed. A Monte Carlo calculation was used to evaluate uncertainties obtained from these nonlinear fitting calculations. It was found that the 1-D linear arrays constrained the resulting current profile roughly as effectively as the conventional MSE measurement. At least 5 or more set of 1-D imaging arrays were required to sufficiently sample the off-midplane plasma region.

The bulk of the modeling concentrated on the 2-D imaging array. Here, it was shown that the full $\mathrm{q}(\mathrm{r})$ profile can be recovered to within an error of $\leq 10 \%$ for $\mathrm{q}(0)$ and $<5 \%$ over most of the plasma cross-section with a noise level of $1 \%$ in the measured intensity profiles, showing that the SXR imaging provides a very strong constraint to the q-profile. Better sensitivity is obtained at high bt. For example with bt $\sim 25 \%$ sq $\sim 3 \%$ over most of the plasma cross-section. Thus, this measurement tehnique is especially powerful for low-field, high bt, ST plasmas, where conventional MSE and poalrimetry measurements are especially problematic. Assuming sufficient accuracy in the imaging system calibration, obtaining the requisite noise level of $1 \%$ due to photon statistics is readily achievable in large fusion-grade plasmas. In general, high-performance, high-beta ST plasmas show higher sensitivity to deducing $\mathrm{q}(\mathrm{r})$ from the image data than do larger aspect ratio and/or lower beta plasmas.

\section{B) Soft X-ray Camera Development}

Two generations of designs for a tangentially viewing 2-D SXR imaging system were developed for tests of this concept on the Pegasus ST experiment. A prototype SXR pinhole camera imaging system consisted of a pinhole imaging aperture, a large scintillator screen, and a commercial intensified video camera which viewed the scintillator from the rear (i.e., using a transmissive scintillator). This prototype SXR camera was installed on Pegasus with a pinhole imaging system with a 0.1 micron thick Be filter and large $(20 \mathrm{~cm}$ diameter) scintillator screen (type 43 - Gd2O2S:Tb). This system operated with a PC and frame-grabber data acquisition system to provide first SXR images from low current Pegasus discharges during an early experimental campaign. While this system was much less sensitive than the final camera design described below, it provided the first tests of the available signals in lowpower Pegasus plasmas.

Soft X-ray images of the interior of the plasmas on Pegasus were obtained in the Fall 2000 campaign. Since the plasma current and temperature were low compared to the eventual full-power operation for Pegasus, the resultant images, integrated over a $5 \mathrm{msec}$ period, had only modest signal-tonoise. Nevertheless, they were useful in constraining the central $q(0)$ in magnetic reconstruction of Pegasus plasmas for the first time.

A more sensitive first-generation SXR CCD imaging system, complete with data acquisition computer and interfacing hardware, was then assembled and tested for integration into the SXR camera for reasonable equilibrium measurements. This approach was tested on Pegasus as a simple SXR camera to debug control, acquisition, and noise issues. Major changes were incorporated into this final design of the large SXR camera assembly for Pegasus applications. A large-area (40 $\mathrm{mm}$ diameter) 
microchannel plate intensifier with intrinsic gating capability for fast shuttering was obtained. Most importantly, the design was converted to a reflection-imaging geometry to provide an additional increase of a factor of 5 over that expected from the previous in-line illumination design under consideration. This system used a $0.5 \mathrm{~mm}$ diameter pinhole and an Gd2O2S:Pr phosphor for faster time response. A scientific-grade CCD camera was used to optimize the signal-to-noise. This redesign required the development of a folded optics vacuum enclosure for the SXR stage (c.f., Fig. 1). The required large vacuum housing with reflection-imaging capability was installed on a dedicated port on Pegasus. A multiple Be-filter selection assembly was incorporated into the system design to provide sensitivity to a wider range of plasma temperatures without breaking vacuum. Both $\mathrm{Be}$ and $\mathrm{Ni}$ foil filters were used, and $\mathrm{Ni}$ was found to be more effective in rejecting the strong edge emissions in the relatively cold Pegasus plasmas.

An in-lab calibration procedure and apparatus was assembled. The apparatus consisted of an electron-beam X-ray line source with a photon-counting proportional counter as a calibration standard, which will allowed tests of camera sensitivity and uniformity in the lab, and allowed testing of an ultraviolet Hg-lamp transfer standard for in-situ calibration of the camera uniformity. White field calibrations and geometry calibrations were performed with both visible light and a Fe-55 X-ray calibration source. The Fe-55 source was used to measure absolute response while avoiding the continuum interference that occurs with the e-beam source.

Finally, calculation of model images from realistic plasmas with the relatively wide-angle viewing geometry necessary on a low-aspect ratio experiment showed that the large angles of incidence on the Be filters did not significantly distort the resulting images. Any residual effects of these large incidence angles on the resultant plasma equilibrium reconstruction did not appear significant above the measured noise levels. These findings were useful in that they showed that simple flat Be filters could be used instead of much more complex and expensive shaped filter assemblies.

\section{C) Scientific Results}

The first-generation SXR imaging system was installed and operated on the Pegasus ST experiment. It was used to measure moderate-current plasmas, Ip $\sim 150 \mathrm{KA}$, which had high density but relatively low electron temperatures (few $100 \mathrm{eV}$ ). Since these gave relatively low signal levels, some shots were averaged together to both improve the photon statistics and average over any residual MHD behavior. The image noise was in the $2-4 \%$ range rather than the more desired $1 \%$ target range.

For a modestly shaped ST plasme (i.e., $\mathrm{A}=1.17, \mathrm{~K}=1.4$ ), reasonably good matching between the model and measured intensity contours was obtained over most of the plasma interior (Fig. 2). The random uncertainty in the measured $\mathrm{q}(\mathrm{r})$ profile was $\sim 13 \%$ for $\mathrm{q}(0)$ and dropped down to $<5 \%$ for the outer $2 / 3$ of the plasma cross-section. The magnetic reconstructions with the SXR image as an additional constraint, in concert with the usual external magnetics, showed markedly different profiles from those derived with the external magnetics only, as shown in Fig. 3. However, the reconstructions obtained with the imaging constraints included produced more physical results. The external magnetics measurements indicated an oscillatory current profile and extremely peaked pressure profile, with $\mathrm{q}(0)$ $\approx 1$. In contrast, addition of the internal image data produced ahollow but relatively monotonic $\mathrm{j}{ }^{\circledR}$ profile, and a flat interior $\mathrm{q}{ }^{\circledR}$ profile with $\mathrm{q}(0) \sim 2$. The latter profile is more consistent with the observation a large $\mathrm{m} / \mathrm{n}=2 / 1$ internal tearing mode existing over most of the plasma cross section.

In addition, a hollow current profile was found to be correlated with a fast current ramp, as expected. The q-profile reconstructions were also consistent with the presence or absence of large-scale MHD activity. In particular, a very flat internal current profile was obtained for discharges with large internal islands. A comparison of the deduced $q(r)$ profile with those obtained using conventional power-law profiles using external magnetics only indicted that the internal profiles were flatter than 
allowed by the usual models.

The tangential image contour measurements were found to constrain the $q(r)$ profile reconstruction to an uncertainty of $<14 \%$ in the plasma center, with improving accuray at larger minor radii, This result is consistent with the attained image noise of $2-4 \%$. A higher signal-to-noise ratio will tighten the image constraint, and the model result of $\mathrm{sq}<10 \%$ error in $\mathrm{q}(0)$ should be attainable with this diagnostic technique.

In conclusion, these result represent the first measurements of the current profile in a large spherical torus plasma. It was found that the measurement of the flux surface shape through SXR imaging provides a useful constraint on the internal plasma profile reconstruction. Tangential X-ray intensity images can provide adequate constraint on the q-profile with relative image noise $<1 \%$ or so. These results appear encouraging enough to consider deployment on NSTX or DIII-D to compare and contrast with other $\mathrm{q}(\mathrm{r})$ measurements.

Much of this work constituted a graduate student $\mathrm{PhD}$ thesis project.

\section{D) Future Direction}

Finally, a next-generation SXR 2-D imaging camera design was studied in concept. Basically, it uses direct illumination of a back-thinned CCD imaging detector for direct conversion of the X-rays into charge collected on the array. Extensive modeling shows this to be a promising approach to a more compact, mode sensitive SXR imaging system for current profile measurements, if the requisite largearea CCD camera is made available. Time resolution of the plasma measurements appears feasible using a 2-D masking system that allows local frame storage on the the CCD chip. Fabrication and deployment of this concept was beyond the scope of this grant, but its basic design considerations were developed under this study.

\section{Publications}

"Application of X-ray imaging to Current Profile Measurements in the Pegasus Experiment", K. Tritz, R. Fonck, and T. Thorson, Rev. Sci. Intrum. 70, 595 (1999).

"Current Profile Reconstruction Using X-ray Imaging on the Pegasus Toroidal Experiment", K. Tritz, Ph.D. Thesis, University of Wisconsin-Madison (2002).

"Tangential Soft X-ray Imaging for Shape and Current Profile Measurements", K. Tritz, R. Fonck, M. Reinke, and G. Winz, Rev. Sci. Intrum. 74, 2161 (2003).

"Design and Noise Model for CCD-based, Time-resolved PHA Measuremetns", R. Schooff, S. Diem, R. Fonck, M. Reinke, and K. Tritz, Rev. Sci. Intrum. 74, 2007 (2003).

"Current Profiles in the MEDUSA and Pegasus Spherical Torus Experiments", G. Garstka, R, Fonck, and K, Tritz, to be submitted to Nuclear Fusion. 

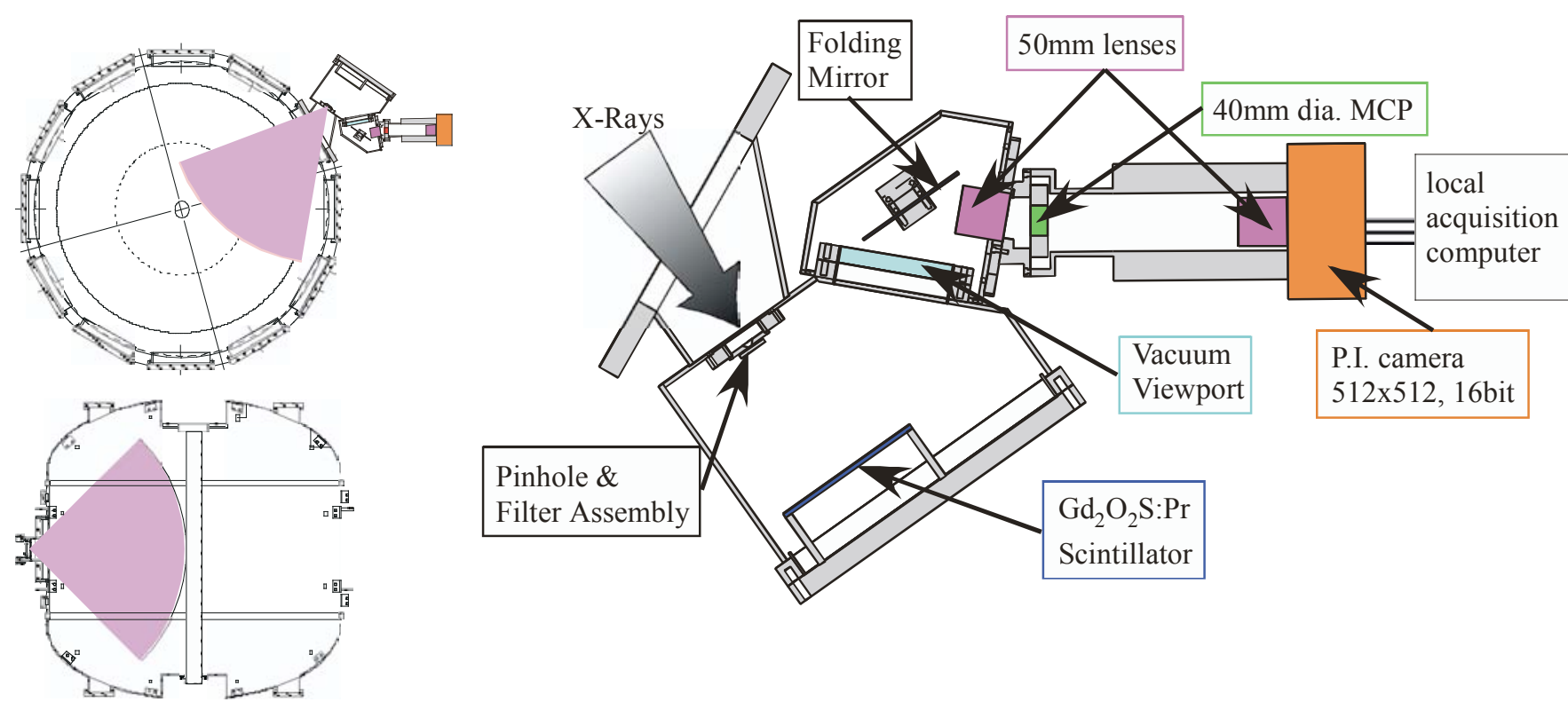

Figure 1. Left: horizontal and vertical viewing sightlines of the SXR imaging system on the Pegasus experiment. Right: schematic of the 1st generation SXR imaging system with folded optical train and reflective phosphor imaging system.
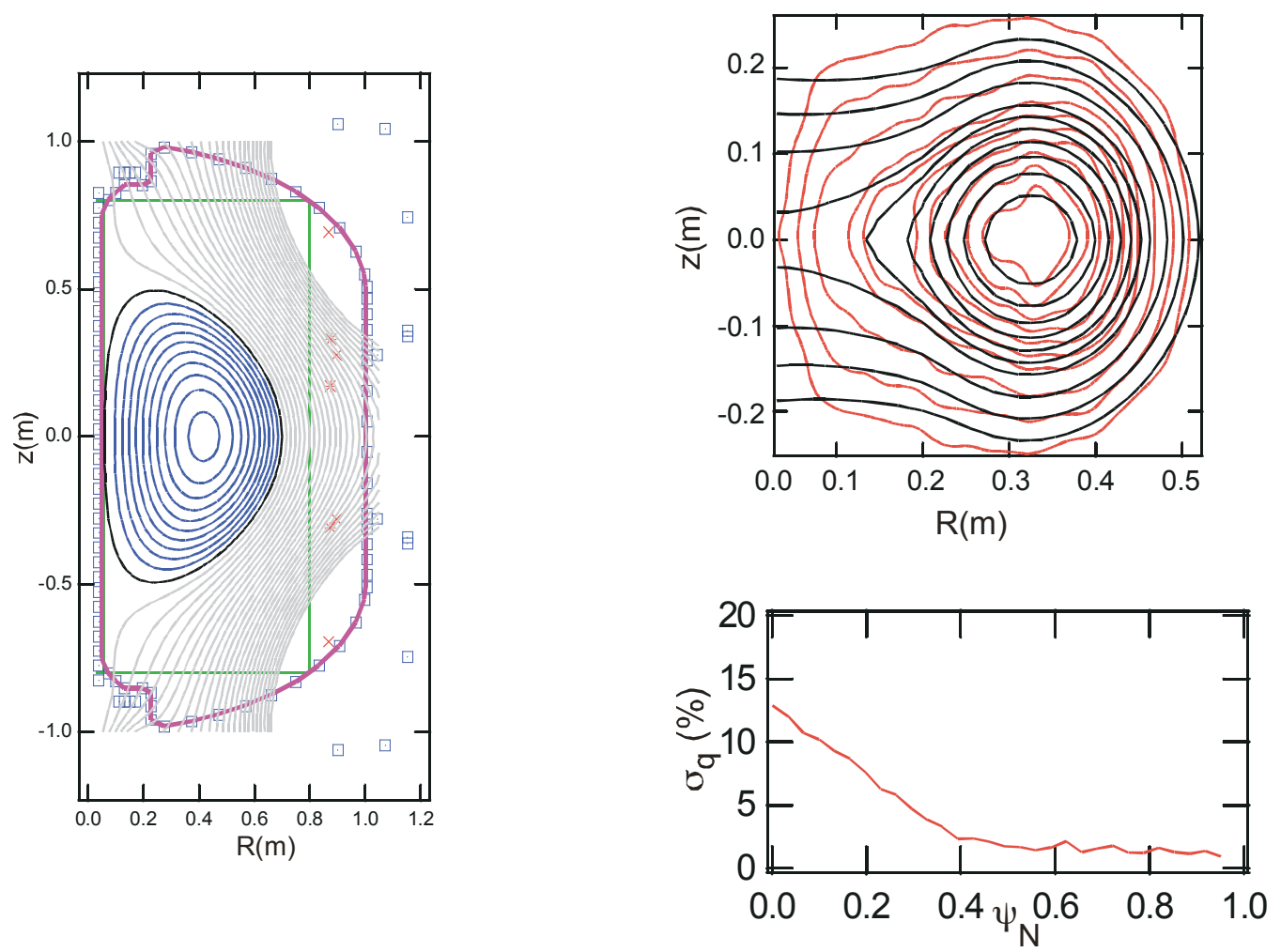

Figure 2. Results of reconstructed plasma equilibrium with added constraint of SXR image fitting. a) 

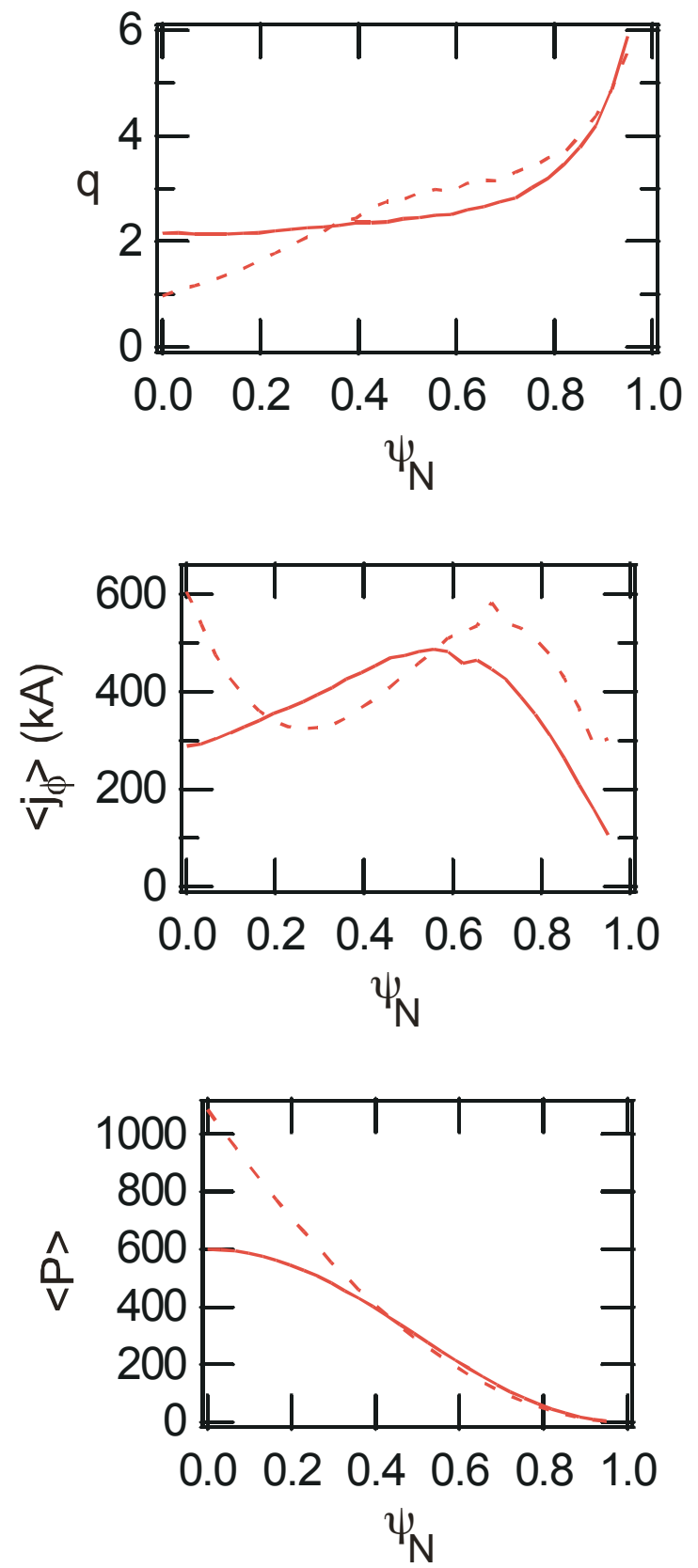

\begin{tabular}{l}
\multicolumn{3}{c}{ Parameter Comparison } \\
\begin{tabular}{|l|l|c|}
\multicolumn{1}{c}{ Spline } & Ext. Mag. \\
\hline $\mathrm{I}_{\mathrm{p}}$ & $135 \mathrm{kA}$ & $144 \mathrm{kA}$ \\
$\beta_{\mathrm{T}}$ & $0.1+/-0.02$ & 0.08 \\
$\mathrm{I}_{\mathrm{i}}$ & $0.43+/-0.06$ & 0.34 \\
$\mathrm{q}_{0}$ & $2.2+/-0.3$ & 1.0 \\
$\mathrm{q}_{95}$ & $5.9+/-0.4$ & 5.6 \\
$\mathrm{~A}$ & $1.21+/-0.02$ & 1.24 \\
$\kappa$ & 1.5 & 1.6 \\
\hline
\end{tabular}
\end{tabular}

Figure 3. Comparison of plasma profiles deduced with internal SXR imaging measurements and spline functional fits included in magnetic reconstruction (solid lines) to those produced with external magnetics only (dashed lines). 